\title{
Catherine Horel, De l'exotisme à la modernité. Un siècle de voyage français en Hongrie (1818-1910)
}

Daniel Baric

\section{(2) OpenEdition}

1 Journals

Édition électronique

URL : https://journals.openedition.org/austriaca/1536

DOI : 10.4000 /austriaca. 1536

ISSN : 2729-0603

Éditeur

Presses universitaires de Rouen et du Havre

\section{Édition imprimée}

Date de publication : 1 juin 2020

Pagination : 254-258

ISBN : 979-10-240-1492-0

ISSN : 0396-4590

\section{Référence électronique}

Daniel Baric, "Catherine Horel, De l'exotisme à la modernité. Un siècle de voyage français en Hongrie (1818-1910)», Austriaca [En ligne], 90 | 2020, mis en ligne le 01 juin 2020, consulté le 12 octobre 2022 URL : http://journals.openedition.org/austriaca/1536 ; DOI : https://doi.org/10.4000/austriaca.1536

Ce document a été généré automatiquement le 12 octobre 2022.

Tous droits réservés 


\title{
Catherine Horel, De l'exotisme à la modernité. Un siècle de voyage français en Hongrie (1818-1910)
}

\author{
Daniel Baric
}

\section{RÉFÉRENCE}

Catherine Horel, De l'exotisme à la modernité. Un siècle de voyage français en Hongrie

(1818-1910), Montrouge, Éditions du Bourg, 2018, 226 p., ISBN 978-2-490650-01-9, $24 €$.

1 Parue à Budapest en 2004 sous le même titre, l'étude de Catherine Horel est publiée dans une version revue et actualisée. La chronologie prise en compte couvre près d'un siècle, entre le voyage du savant François-Sulpice Beudant, auteur d'un Voyage minéralogique et géologique en Hongrie pendant l'année 1818 (Paris, 1822) et celui de Pierre Marge, auteur d'un Voyage en automobile dans la Hongrie pittoresque (Paris, 1910) effectué en compagnie du maire de Lyon Édouard Herriot. L'étude met en relief l'évolution des modes de transport, qui passent de la chaise de poste à l'automobile, reflétant le passage à une forme de voyage qui n'est plus celle de l'exploration. En d'autres termes, la Hongrie perd progressivement son qualificatif de terra incognita, parée d'un imaginaire de terre d'aventure, de contrée encore vaguement dangereuse, qui, bien que proche, étonne encore par sa singularité.

2 Au cours de ce siècle de voyages, la révolution de 1848 apparaît comme une césure majeure. Les rares voyageurs français qui s'aventurèrent en pays hongrois durant cette période furent, à l'instar du couple Blaze de Bury, favorables aux Autrichiens. Le retour des voyageurs français après le Compromis de 1867 et surtout l'exposition universelle de Vienne de 1873 est prolongé par la découverte de Budapest comme métropole moderne. L'exploration des campagnes se mue, par conséquent, en une recherche des dernières traces d'exotisme. Dans l'abondante bibliographie des récits de voyage, ce distinguo entre la capitale et la province est très général, alimenté sans doute par des 
auteurs soucieux de donner à lire ce qu'ils supposent devoir être l'attente de leurs lecteurs.

3 Les quelque 70 auteurs qui forment le corpus étudié ont des statuts assurément divers, les uns ayant bénéficié d'une notoriété plus importante que d'autres. Par ailleurs, peu de grandes plumes, et d'une manière générale peu de femmes, produisirent des récits de voyage en Hongrie. Même pour les auteurs en vue qui se rendirent en Hongrie à cette époque, comme ce fut le cas de Prosper Mérimée, nous ne disposons que de mentions dans sa correspondance. Des documents inédits complètent le panorama. Les récits sont présentés dans une évolution à la fois historique et thématique. Par petites touches se forme une mosaïque d'impressions : survivance des costumes folkloriques chez les peuples non magyars, découverte de la diversité religieuse en contraste avec l'Autriche catholique. C'est un aperçu sur la Hongrie historique qui s'offre ainsi, incluant le Banat, la Transylvanie et la Voïvodine.

4 Catherine Horel propose une typologie des voyageurs: elle distingue les savants, universitaires ou autres, qui sillonnèrent le pays, par contraste avec nombre de récits écrits par des auteurs qui citent rarement les ouvrages consultés en amont de leur itinéraire. Nombreux sont ceux qui comptent sur l'obligeance de leurs hôtes pour leur expliquer la situation qu'ils rencontrent sur place. La méconnaissance généralisée du hongrois entraîne le passage systématique par l'allemand ou le français, voire le latin, pour la communication. Cet outillage linguistique réduit explique le peu de contacts avec les minorités, slaves ou roumaines en particulier, dont les langues sont tout aussi peu maîtrisées. Cela génère certaines approximations, relevées par les contemporains hongrois. On reproche ainsi à Victor Tissot, auteur d'un Voyage au pays des Tsiganes (Paris, 1881), l'identification hâtive entre Hongrois et Tsiganes. Il leur aurait pourtant été possible de faire appel à des guides bien informés couvrant l'ensemble des territoires habsbourgeois, qui commencèrent à paraître en français à partir des années 1850, souvent sous forme de traductions de l'allemand (guides Baedeker), mais pas exclusivement (guides Joanne chez Hachette).

Comme le souligne l'auteur, la lecture de ces récits permet de resituer le débat récurrent sur la situation relative de développement de la Hongrie au XIX ${ }^{e}$ siècle. Les débats historiographiques actuels sur le degré relatif de développement de l'AutricheHongrie tendent à relativiser la notion d'arriération économique qui lui fut longtemps attachée : vers 1900, le réseau ferré hongrois construit en étoile autour de Budapest atteint une densité comparable à la situation française. De plus, l'appréhension d'un différentiel économique n'est pas le même suivant le sens géographique du déplacement : en venant du nord, du sud ou de l'est, la Hongrie paraît de beaucoup moins en retard de développement que pour le visiteur qui s'y rend depuis l'ouest. Ainsi, Pierre Marge trouve peu d'automobilistes en dehors de l'axe viennois et déplore l'état des routes en sortant de cette voie: il est vrai que la première automobile à rentrer dans Budapest en 1895, une Benz, était en provenance de Vienne. Le Danube représente également un axe de pénétration privilégié de l'espace hongrois, qu'il permet de rejoindre assez rapidement depuis Vienne, même si, en 1823, il faut tout de même compter 23 heures pour rejoindre la capitale hongroise depuis le centre impérial. À mesure que le train détrône la navigation fluviale au début $d u x^{e}$ siècle, la rapidité est de plus en plus recherchée par les voyageurs, qui ne se laissent plus charmer par la lenteur. 
6 L'ouvrage aborde toutes ces questions de transport et de perception de l'espace autour de trois chapitres thématiques: la permanence de certains clichés, l'émergence de Budapest comme métropole d'Europe centrale et le «miroir politique » que la Hongrie représente, en tant que reflet d'une lecture française de la présence germanique dans l'Europe danubienne. Suivant l'origine géographique des voyageurs, certains clichés perdurent, les Parisiens affichant une certaine supériorité à l'égard de la Hongrie, ce qui n'est pas le cas des provinciaux. Ainsi, une manière de voir les Hongrois comme des êtres que les Autrichiens ont bien du mal à discipliner se retrouve sous la plume de nombreux voyageurs.

7 En filigrane se pose la question de la perméabilité de la Hongrie aux influences occidentales et orientales. Alors qu'à Bude " une odeur de bureaucratie autrichienne » oppresse Hippolyte Durand, auteur de l'ouvrage Le Danube allemand et l'Allemagne du Sud (Tours, 1863), le bois de la ville de Pest (Város liget) est décrit comme un «Bois de Boulogne oriental » et Xavier Marmier ressent dans le pays le «souffle voluptueux de l'Orient » en 1847. La Hongrie est perçue comme le reflet d'autres réalités, ce qui est indicatif de la difficulté des voyageurs français à cerner une civilisation qui leur demeure fondamentalement étrangère. En point d'orgue de ces voyages au cours du $\mathrm{XIX}^{\mathrm{e}}$ siècle se trouvent deux manifestations d'ampleur internationale: l'exposition nationale de 1885 , dont une délégation de journalistes et d'écrivains rendit compte, ainsi que les fêtes du millénaire de 1896 qui marquent, comme l'indique le chapitre central de l'ouvrage, «Le Triomphe de Budapest ». Ce sont les moments d'une prise de conscience d'un rattrapage hongrois et d'une véritable altérité. La francophilie que les voyageurs découvrent apparaît comme le gage d'un potentiel anti-autrichien prometteur. Ainsi, comme l'écrit Charles-Louis Chassin dans La Hongrie. Son génie et sa mission (Paris, 1856), les Hongrois apparaissent comme la preuve que «cet immoral amalgame que l'on nomme la monarchie autrichienne » n'est en rien naturel. L'intérêt pour la Hongrie apparaît donc de plus en plus au cours de ce siècle de voyage français comme le résultat d'une motivation avant tout anti-germanique. Une assimilation de l'Autriche à la Prusse est faite à cette occasion, ignorante du fait que la Hongrie ellemême penche de plus en plus vers l'Allemagne. La francophilie hongroise est interprétée comme prémisse d'un renversement des alliances, qui aurait pour corollaire le détachement de la Hongrie du bloc germanique. C'est également à l'aune de ces considérations purement françaises que peut se lire un regard favorable ou non aux Hongrois et, par contraste, aux Slaves (p. 93).

8 Quelques précisions complémentaires sur le profil des voyageurs auraient sans doute permis de déterminer ce qui, chez tel "écrivain", à l'instar de Jacques Boucher de Perthes (p. 71), peut être lu comme le reflet d'une fonction sociale (directeur des douanes) ou scientifique (en tant qu'éminent préhistorien). S'il faut bien avoir à l'esprit parmi les contributeurs français ce que signifie l'affiliation politique, la provenance géographique pour cerner la fabrique du discours, les quelques auteurs francophones, mais d'origine suisse, auraient peut-être mérité une analyse en soi : y a-t-il en effet un regard spécifiquement helvétique, genevois, fribourgeois ou bien calviniste sur la Hongrie? Les spécificités françaises de la perception des réalités hongroises dans ce siècle pourraient de la sorte apparaître de manière plus systématique. Ainsi, il semble avéré que chez les auteurs français se soit manifesté un intérêt moindre pour le développement économique et ses mécanismes que dans les récits produits par les Anglais en Hongrie. Un approfondissement des questions d'imagologie et de 
chronologie de ces images permettrait sans doute de situer ce discours français sur la Hongrie dans le contexte européen, en puisant notamment dans les travaux de Sylvain Venayre sur la question du voyage européen au XIX ${ }^{e}$ siècle ou dans les réflexions sur les voyageurs germanophones de Mirna Zeman ${ }^{1}$ pour la période précédente, qui valide une approche régionale. Ainsi, les remarques de l'industriel Pierre Marge en Hongrie gagneraient à être comparées avec ce qu'il observe lors d'un itinéraire motorisé à travers les Balkans autrichiens (1905) et en Espagne (1909), deux autres périphéries européennes.

On pourra regretter que les signes diacritiques ne soient pas toujours parfaitement rendus (Köbanya pour Kőbánya, p. 160) et que les illustrations, belles vignettes insérées dans le texte, soient parfois difficilement exploitables en raison de leurs format et définition. L'absence d'index géographique est également dommageable à une utilisation plus intensive de cette recherche. Mais l'ouvrage présente nombre de textes qui, par leur juxtaposition et leur analyse, apportent une grande richesse d'aperçus. La découverte dans la Grande Plaine de "villes non urbaines » par l'agronome René Gonnard (p. 43) ou l'observation par Elisée Reclus en 1872 de la persistance de ruines du conflit de 1848 près d'un quart de siècle après les événements, par exemple, apportent un regard précieux pour saisir au plus près une réalité qui forme l'arrière-plan de toute réflexion sur le $\mathrm{XIX}^{\mathrm{e}}$ siècle, en l'occurrence franco-hongrois et plus largement habsbourgeois.

\section{NOTES}

1. Mirna Zeman, Reise zu den "Illyriern». Kroatienstereotype in der deutschsprachigen Reiseliteratur und Statistik (1740-1809), Munich, Oldenbourg, 2013. 